\title{
Modified Gradient Flow Method for Solving One-Dimensional Optimal Control Problem Governed by Linear Equality Constraint
}

\author{
Olusegun Olotu ${ }^{\mathrm{a}}$, Charles Aladesaye ${ }^{\mathrm{b}}$, Kazeem Adebowale Dawodu, ${ }^{\mathrm{a}, *}$ \\ ${ }^{a}$ Department of Mathematical Sciences, The Federal University of Technology Akure, Nigeria \\ ${ }^{b}$ Dept. of Mathematics, School of Science, College of Education, Ikere-Ekiti, Ekiti State, Nigeria
}

\begin{abstract}
This study presents a computational technique developed for solving linearly constraint optimal control problems using the Gradient Flow Method. This proposed method, called the Modified Gradient Flow Method (MGFM), is based on the continuous gradient flow reformulation of constrained optimization problem with three-level implicit time discretization scheme. The three-level splitting parameters for the discretization of the gradient flow equations are such that the sum of the parameters equal to one $\left(\theta_{1}+\theta_{2}+\theta_{3}=1\right)$. The Linear and quadratic convergence of the scheme were analyzed and were shown to have first order scheme when each parameter exist in the domain [0, 1] and second order when the third parameter equal to one. Numerical experiments were carried out and the results showed that the approach is very effective for handling this class of constrained optimal control problems. It also compared favorably with the analytical solutions and performed better than the existing schemes in terms of convergence and accuracy.
\end{abstract}

DOI:10.46481/jnsps.2022.589

Keywords: Optimal Control, Gradient Flow, three-level splitting parameters, discretization scheme, linear and quadratic convergence

Article History :

Received: 15 January 2022

Received in revised form: 23 February 2022

Accepted for publication: 23 February 2022

Published: 28 February 2022

(c)2022 Journal of the Nigerian Society of Physical Sciences. All rights reserved. Communicated by: J. Ndam

\section{Introduction}

Many authors, in literature, have attempted the class of linearly constrained optimal control problems using the classical analytical techniques of the Hamilton-Jacobi-Bellman (HJB) or "Pontryagin Maximum Principle" by Pontryagin et al. in [14]; though this approach may proof difficult in some cases when inclusive of non-differentiable delay terms. The numerical approach has also been fully handled by several authors like Betts

${ }^{*}$ Corresponding author tel. no: +2348032541380

Email address: dawodukazeem@futa.edu.ng (Kazeem Adebowale Dawodu) in [4], Olotu and Dawodu in [11, 12], Adekunle [1] and Akeremale [2], using the direct numerical methods. This method deploys the "first discretized - then - optimize" technique which is known for its amenability to well-structured discretization schemes and optimization solvers after the unconstrained formulation of the control problem using any of the penalty function methods or method of multipliers. The direct method of nonlinear programming formulation tends to eradicate the problem of ill-conditioning associated with control problems with delay dynamical systems.

However, this research paper seeks for a better approach in the 
formulation of the control operators in the optimization of the discretized optimal control problem. The gradient flow method was first applied to nonlinear programming problems by Evtushenko [5] and later improved by Evtushenko and Zhadan [6]; while the application to unconstrained problems was by Behrman [3] and the unified approach to nonlinear optimization problem was by Wang et al. [15]. The unified gradient flow algorithm exhibits linear convergence for $\theta \in[0,1)$ and quadratic convergence at $\theta=1$. As an improvement over [15], a modified gradient flow method MGFM was proposed using the gradient formula for Lagrange multiplier formulation. The development of this method requires a continuous gradient flow approach based on a three level implicit time discretization scheme with splitting parameter $\theta_{1}, \theta_{2}$ and $\theta_{3}$. The convergence of the solution of the modified discretized gradient flow equation to a local minimum of the original problem, either linearly or quadratically depending on the choice of $\theta$, will as well be demonstrated. However, the technique can also be adapted to handle inequality constraint problems by introducing slack variables. Three test problems were solved and their numerical results presented in comparison with existing methods. This is to demonstrate the effectiveness of the method in solving constrained nonlinear programming problems with either continuous variables or a mixture of continuous and discrete variables.

The structure of this paper is as follows: Section 3 is dedicated to the idea of gradient flow approach for unconstrained optimization while Section 4 presents the modified gradient flow algorithm for constrained optimization in Section 2 using the 3level splitting parameters and as well as the convergence analysis. The Algorithm was experimented on two numerical examples and comparison made with the Conjugate Gradient Method (CGM) in Section 4.

\section{Statement of problem}

The general form of the One-Dimensional Optimal Control Problem is given as

$$
\begin{gathered}
\text { Minimize } J(x, u)=\int_{t_{0}}^{T} F(t, x(t), u(t)) d t, \\
\text { Subject to } \dot{x}(t)=f(t, x(t), u(t)), t \in\left[t_{0}, T\right], \\
x\left(t_{0}\right)=x_{0},
\end{gathered}
$$

where $x(t) \in C^{1}\left[t_{0}, T\right] \in \mathbb{R}, u(t) \in L^{2}\left[t_{0}, T\right] \in \mathbb{R}, F:\left[t_{0}, T\right] \times$ $\mathbb{R} \times \mathbb{R} \rightarrow \mathbb{R}$ and $f:\left[t_{0}, T\right] \times \mathbb{R} \times \mathbb{R} \rightarrow \mathbb{R}$.

Thus, $x(t)$ is the state variable which describes the state of the system at any point in time and $u(t)$ is the control variable in the optimization problem. However, for linear-quadratic problem, the objective functional is written as $F(t, x(t), u(t))=\left(p x^{2}(t)+\right.$ $\left.q u^{2}(t)\right)$ while the linear differential constraint is written as $f(t, x(t), u(t))=x^{k+1}:=(I+h \nabla g)^{-1}(I-h \nabla f) x^{k}$, $a x(t)+b u(t)$, where $p, q, a, b \in \mathbb{R}$ and $p, q>0$.

\section{Research Methodology}

This paper considered both the theoretical and numerical analyses of the problem in the development of the algorithm for solving eqns. (1) to (3). The discrete form of the quadratic objective functional and the linear differential constraint are constructed using the Trapezoidal and Euler method respectively. The recurrence relation for both the objective functional and constraint were developed to help construct their respective matrix operators. Furthermore, the symmetry and positive definite properties of the matrix operators were analyzed to guarantee their consistency and invertibilty in the developed MGFM.

\subsection{Preamble}

Let $f: X \rightarrow \mathbb{R}$ be a functional such that the solution $x(t)$ is a minimizer of $f$. The gradient flow method, with an initial point $x(0) \in X$, seeks to find a minimizer of $f$ by the curve $x(t)$ defined by the differential equation

$$
\frac{d x(t)}{d t}=-\nabla f(x(t)), \quad x(0)=x_{0},
$$

where $\nabla f$ is called the gradient of $f$ and the solution is the called the integral curve such that at each instance proceeds in the direction of the steepest descent of $f$. In an unconstrained optimization problem, the equilibrium points of the gradient flow are the zeros of $\nabla f$, which are exactly the minimizers of $f$. The gradient flow solves the problem $\min f(x), x(0)=x_{0}$ such that in every trajectory $x(t)$ of the gradient flow, then $f(x(t)) \rightarrow$ $p^{*}$ for $p^{*}$ is the minimizer of $f$.

For clarity, the derivative $x^{\prime}(t)$ is descretized using the forward Euler step which yields

$$
x^{k+1}:=x^{k}-h \nabla f\left(x^{k}\right)
$$

or the backward discretization scheme which yields

$$
x^{k+1}:=x^{k}-h \nabla f\left(x^{k+1}\right) .
$$

The backward Euler method can be applied for the numerical integration of gradient flow differential equation, also known as the Proximal minimization method expressed thus:

$$
x^{k+1}:=\operatorname{Prox}_{h, f}\left(x^{k}\right) .
$$

However, the proximal gradient flow algorithm can be interpreted as gradient flows,

$$
\frac{d x(t)}{d t}=-\nabla f(x(t))-\nabla g(x(t))
$$

where $g$ is differentiable. Using the forward-backward integration of the gradient flow, eqn. (7) can be expressed as

$$
x^{k+1}=x^{k}-h \nabla f\left(x^{k}\right)-h \nabla g\left(x^{k+1}\right),
$$


where the forward-backward splitting method above numerically integrates the gradient flow differential equations.

The time-stepping discretization of eqns. (5) and (6) is expressed as follows:

$$
x^{k+1}:=x^{k}-h\left[(1-\theta) \nabla f\left(x^{k}\right)+\theta \nabla f\left(x^{k+1}\right)\right],
$$

where $\theta \in[0,1]$ is a parameter. When $\theta=0$, the above discretization is the explicit Euler's scheme and on the other hand, the implicit backward Euler when $\theta=1$.

\subsection{Discretization of the Objective function}

The discretization of the objective function in eqn. (1) above, the Trapezoidal rule will be used given as;

$$
\int_{t_{0}}^{T} f(x) d x=\frac{h}{2} \sum_{i=0}^{N-1}\left[f\left(x_{i}\right)+f\left(x_{i+1}\right)\right]
$$

Expanding eqn. (9) above, yields;

$$
J(x, u) \approx \frac{h}{2} p \sum_{i=0}^{N-1}\left(x_{i}^{2}+x_{i+1}^{2}\right)+\frac{h}{2} q \sum_{i=0}^{N-1}\left(u_{i}^{2}+u_{i+1}^{2}\right),
$$

where for $t_{i}=t_{0}+i h$ then $x\left(t_{i}\right) \approx x\left(t_{0}+i h\right)=x_{i}, u\left(t_{i}\right) \approx$ $u\left(t_{0}+i h\right)=u_{i}$ for $i=1,2, \ldots, N$ and $N=\left(T-t_{0}\right) / h$. Rearranging eqn. (10) into matrix form yields;

$$
\text { Minimize } \quad J(Z)=\frac{1}{2} Z^{T} M Z+C,
$$

where $Z=\left(x_{1}, x_{2}, \ldots x_{N}, u_{0}, u_{1}, \ldots, u_{N}\right)^{T} \in \mathbb{R}^{2 N+1}, C=\frac{1}{2} x_{0}^{2} h p \in$ $\mathbb{R}$ and $M \in \mathbb{R}^{(2 N+1) \times(2 N+1)}$ is a $(2 N+1) \times(2 N+1)$ matrix operator with diagonal entries $m_{i j}$ as expressed below:

$$
\left[m_{i j}\right]=\left\{\begin{array}{lcc}
2 h p, & 1 \leq i \leq N-1, & j=i, \\
h p, & i=N, & j=i \\
h q, & i=N+1,2 N+1, & j=i, \\
2 h q, & N+2 \leq i \leq 2 N, & j=i \\
0, & \text { elsewhere }
\end{array}\right.
$$

\subsection{Discretization of the Constraint function}

The differential constraint $\dot{x}(t)=f(t, x(t), u(t))=a x(t)+$ $b u(t)$ was discretized by slotting it into the Euler's scheme $x_{i+1}=$ $x_{i}+\dot{x} h$ with $t_{i}=t_{0}+i h$, to arrive at

$$
x_{i+1}=c x_{i}+d u_{i},
$$

where $c=1+h a, d=h b$ and $i=0,1,2, \ldots, N-1$. And upon expansion and re-arrangement of eqn. (13) into matrix form yields $G Z=k$ express as

$$
g(Z)=G Z-k=0,
$$

where $Z$ is of dimension $(2 N+1) \times 1$ and $G$ is of dimension $N \times(2 N+1)$ as shown below;

$$
G=\left[\begin{array}{cccc|ccccc}
1 & 0 & \cdots & 0 & -d & 0 & \cdots & \cdots & 0 \\
-c & 1 & \ddots & \vdots & 0 & -d & 0 & \ddots & \vdots \\
0 & \ddots & \ddots & 0 & \vdots & \ddots & \ddots & \ddots & 0 \\
\vdots & \ddots & -c & 1 & 0 & \cdots & 0 & -d & 0
\end{array}\right]
$$

and

$$
k=\left[\begin{array}{c}
c x_{0} \\
0 \\
\vdots \\
0
\end{array}\right]
$$

with the entries defined as follows: $g_{i j}=1$ for $1 \leq i \leq N$ and $j=i ; g_{i j}=-d$ for $1 \leq i \leq N$ and $j=N+i ; g_{i j}=-c$ for $2 \leq i \leq N$ and $j=i-1$ while $g_{i j}=0$ otherwise. Hence, eqns. (11) and (14) give the quadratic representation for the constrained minimization problem as

$$
\text { Minimize } \quad J(Z)=\frac{1}{2} Z^{T} M Z+C,
$$$$
\text { subject to } G Z-k=0 \text {. }
$$

\subsection{Lagrangian formulation of the Constraint problem}

The unconstrained form of the optimal control problem can be obtained using the Lagrangian function on eqn. (15) as stated below:

$$
\begin{aligned}
L(Z, \lambda) & =\frac{1}{2} Z^{T} M Z+C+\lambda^{T}(G Z-k), \\
L(Z, \lambda) & =J(Z)+\lambda^{T} g(Z)
\end{aligned}
$$

where $\lambda=\left(\lambda_{1}, \lambda_{2}, \ldots, \lambda_{N}\right)^{T} \in \mathbb{R}^{N}$ is the Lagrangian multiplier. The pair of points $\left(Z^{*}, \lambda^{*}\right)$ is said to be a stationary pair points of eqn. (17) if the following first order necessary optimality conditions hold:

$$
\begin{aligned}
& L_{Z}\left(Z^{*}, \lambda^{*}\right)=J_{Z}\left(Z^{*}\right)+g_{Z}^{T}\left(Z^{*}\right) \lambda^{*}=0, \\
& L_{\lambda}\left(Z^{*}, \lambda^{*}\right)=g\left(Z^{*}\right)=0,
\end{aligned}
$$

where

$$
\begin{aligned}
L_{Z}(Z, \lambda) & =\left(\frac{\partial L(Z, \lambda)}{\partial x_{1}}, \cdots, \frac{\partial L(Z, \lambda)}{\partial u_{N}}\right)^{T}, \\
L_{\lambda}(Z, \lambda) & =\left(\frac{\partial L(Z, \lambda)}{\partial \lambda_{1}}, \cdots, \frac{\partial L(Z, \lambda)}{\partial \lambda_{N}}\right)^{T}, \\
J_{Z}(Z) & =\left(\frac{\partial J(Z)}{\partial x_{1}}, \cdots, \frac{\partial J(Z)}{\partial u_{N}}\right)^{T},
\end{aligned}
$$

denote the gradient vectors of $L$ and $J$ with the dimensions $(2 N+1) \times 1, N \times 1$ and $(2 N+1) \times 1$ respectively. Similarly, we 
have the Jacobian of $g$ with dimension $N \times(2 N+1)$ represented below:

$$
g_{Z}(Z)=\left[\begin{array}{cccc}
\frac{\partial g_{1}(Z)}{\partial x_{1}} & \frac{\partial g_{1}(Z)}{\partial x_{2}} & \ldots & \frac{\partial g_{1}(Z)}{\partial u_{N}} \\
\frac{\partial g_{2}(Z)}{\partial x_{1}} & \frac{\partial g_{2}(Z)}{\partial x_{2}} & \ldots & \frac{\partial g_{2}(Z)}{\partial u_{N}} \\
\vdots & \vdots & \vdots & \vdots \\
\frac{\partial g_{N}(Z)}{\partial x_{1}} & \frac{\partial g_{N}(Z)}{\partial x_{2}} & \ldots & \frac{\partial g_{N}(Z)}{\partial u_{N}}
\end{array}\right]
$$

In order to fulfill the above optimality conditions, the continuous gradient flow reformulation with the given initial condition $Z_{0}$ is derived as follows;

$$
\begin{aligned}
& \frac{d Z}{d t}=-L_{Z}(Z(t), \lambda(Z(t))), Z(0)=Z_{0}, \\
& \frac{d Z}{d t}=\varphi(Z) .
\end{aligned}
$$

Substituting eqn. (18) into (21) yields,

$$
\frac{d Z}{d t}=-\left[J_{Z}(Z(t))+g_{Z}^{T}(Z(t)) \lambda^{0}(Z(t))\right], Z(0)=Z_{0}
$$

such that the choice of $\lambda^{0}(Z)$ is to satisfy

$$
L_{Z}\left(Z, \lambda^{0}(Z)\right)=\tau g_{Z}^{T}(Z) g(Z),
$$

in the least square sense, where $\tau>0$ is a parameter.

Substituting eqn. (18) into (24) yields,

$$
J_{Z}(Z)+\lambda^{0}(Z) g_{Z}^{T}(Z)=\tau g_{Z}^{T}(Z) g(Z) .
$$

Obtaining the least squares solution of $\lambda^{0}(Z)$ in en. (25) yields,

$$
\begin{array}{r}
\lambda^{0}(Z) g_{Z}^{T}(Z)=\tau g_{Z}^{T}(Z) g(Z)-J_{Z}(Z), \\
\lambda^{0}(Z)=\left[g_{Z}^{T}(Z)\right]^{\dagger}\left\{\tau g_{Z}^{T}(Z) g(Z)-J_{Z}(Z)\right\},
\end{array}
$$

where the superscript ' $\dagger$ ' refers to the pseudo inverse of $g_{Z}^{T}(Z)$ in Layton [9].

Theorem 3.1. The constraint equations for the discretized optimal control problem $g(Z)$ satisfies the linearly independent constraint qualification (LICQ) for any feasible point $Z^{*}$. The LICQ states that the gradient of the active inequality constraints and the gradient of the equality constraints are linearly independent at $Z^{*}$. eyiu

LICQ holds if $g_{1 Z}(Z), g_{2 Z}(Z), \ldots, g_{N Z}(Z)$ are linearly independent for any feasible point $Z^{*}$. Thus

$$
\alpha_{1} g_{1 Z}(Z)+\alpha_{2} g_{2 Z}(Z)+\ldots+\alpha_{N} g_{N Z}(Z)=0
$$

implies that $\alpha_{i}=0$. Hence, the dimension of the row space of $G$ must be $N$.

Applying the row-reduction process to $G$ in eqn. (3.3), we have $\bar{G}_{*}=[I \mid V]$ written as

$\bar{G}_{*}=$

$$
\left[\begin{array}{cccc|ccccc}
1 & 0 & \cdots & 0 & -d & 0 & \cdots & \cdots & 0 \\
0 & 1 & \ddots & \vdots & -c d & -d & \ddots & \ddots & \vdots \\
\vdots & \ddots & \ddots & 0 & \vdots & \ddots & \ddots & \ddots & \vdots \\
0 & \cdots & 0 & 1 & -c^{N-1} d & \cdots & -c d & -d & 0
\end{array}\right]
$$

where $I \in \mathbb{R}^{N \times N}$ and $V \in \mathbb{R}^{N \times(N+1)}$. The entries $g_{i j}^{*}$ of $\bar{G}_{*}$ can be defined as follows:

$$
\left[g_{i j}^{*}\right]=\left\{\begin{array}{lc}
1, & 1 \leq i \leq N, j=i \\
-c^{k} d, & k+1 \leq i \leq N, j=N+i-2 ; \\
& \text { for } k=1,2, \ldots, N-1, \\
0, & \text { elsewhere. }
\end{array}\right.
$$

Thus, rank of $\bar{G}_{*}$ equals rank of $G$ and $\alpha_{i}=0$ are the parameters that make $g_{1 Z}(Z), g_{2 Z}(Z), \ldots, g_{N Z}(Z)$ linearly independent for any feasible point $Z^{*}$ and $G(Z)$ satisfies LICQ. From theorem (3.1), we have for any feasible point $Z^{*}$ that $\bar{G}_{*} \in \mathbb{R}^{N \times(2 N+1)}$ is re-structured into a $N \times N$ non-singular (invertible) Gram matrix $G_{*}$ given as

$$
G_{*}=g_{Z}(Z) g_{Z}^{T}(Z) .
$$

Multiplying both sides of eqn. (25) by $g_{Z}(Z)$ arrives at

$$
g_{Z}(Z)\left(J_{Z}(Z)+\lambda^{0}(Z) g_{Z}^{T}(Z)\right)=\tau g_{Z}(Z) g_{Z}^{T}(Z) g(Z) .
$$

Therefore, with $G_{*}(Z)=g_{Z}(Z) g_{Z}^{T}(Z)$, eqn. (32) becomes

$$
g_{Z}(Z) J_{Z}(Z)+g_{Z}(Z) \lambda^{0}(Z) g_{Z}^{T}(Z)=\tau G_{*} g(Z)
$$

and simplified to get

$$
G_{*} \lambda^{0}(Z)=\tau G_{*} g(Z)-g_{Z}(Z) J_{Z}(Z) .
$$

Pre-multiplying eqn. (33) by $G_{*}^{-1}$ yields

$$
G_{*}^{-1} G_{*} \lambda^{0}(Z)=G_{*}^{-1} \tau G_{*} g(Z)-G_{*}^{-1} g_{Z}(Z) J_{Z}(Z),
$$

and solving for $\lambda^{0}(Z)$ to obtain

$$
\lambda^{0}(Z)=\tau g(Z)-G_{*}^{-1} g_{Z}(Z) J_{Z}(Z),
$$

where eqns. (27) and (35) are equivalent. Consequently, eqn. (27) can be express as

$$
\begin{aligned}
\lambda^{0}(Z) & =\left(g_{Z}^{T}(Z)\right)^{\dagger}\left[\tau g_{Z}^{T}(Z) g_{Z}(Z)-J_{Z}(Z)\right], \\
& =\tau g_{Z}(Z)-G_{*}^{-1} J_{Z}(Z) g_{Z}(Z),
\end{aligned}
$$

where $G_{*}^{-1}=\left[g_{Z}^{T}(Z) g_{Z}(Z)\right]^{-1}$, hence the pseudo-inverse of $\left[g_{Z}^{T}(Z)\right]$ is $\left[g_{Z}^{T}(Z)\right]^{\dagger}=G_{*}^{-1} g_{Z}(Z)$.

However, eqn. (35) is true when $Z$ is sufficiently close to an equilibrium point $Z^{*}$. The choice of $\lambda^{0}(Z)$ has the advantage of reducing the complexity in the evaluation of the gradient of $\lambda^{0}$ with respect to $Z$. Thus substituting $\lambda^{0}(Z)$ in eqn. (35) above into eqn. (23) yields;

$$
\begin{aligned}
\dot{Z} & =\varphi(Z)=-\left(J_{Z}(Z)+g_{Z}^{T}(Z)[\tau g(Z)\right. \\
& \left.\left.-G^{-1}(Z) g_{Z}(Z) J_{Z}(Z)\right]\right) .
\end{aligned}
$$

In testing the convergence of the gradient flow formulation expressed in eqns. (23) and (24), we then considered the LICQ theorem below. 
Theorem 3.2. Suppose that $\left(Z^{*}, \lambda^{*}\right)$ is a stationary point, then

1. LICQ holds at $Z^{*}$.

2. For any $\bar{Z}$ satisfying $g_{Z}\left(Z^{*}\right) \bar{Z}=0$, then, $\bar{Z}^{T} L_{Z Z}\left(Z^{*}, \lambda^{*}\right) \bar{Z}>0$ where $\bar{Z}=Z-Z^{*}$.

It indicates that the pair $\left(Z(t), \lambda^{0}(Z(t))\right)$ tends to $\left(Z^{*}, \lambda^{*}\right)$ as $t \rightarrow$ $\infty$ provided the starting point $Z_{0}$ is sufficiently close to point $Z^{*}$.

\section{Proof:}

We start by deploying the Poincare-Lyapunov stability theorem stated in Haddad et al. [8] and Morris [10] thus:

Theorem 3.3 (Liapunov Stability). Let $X^{*}$ be an equilibrium point for $X^{\prime}=J(X)$. Let $L: \Omega \rightarrow R$ be a differentiable function defined on an open set $\Omega$ containing $X^{*}$. Suppose further that

1. $L\left(X^{*}\right)=\Omega$ and $L(X)>0$ if $X \neq X^{*}$;

2. $\dot{L} \leq 0$ in $\Omega-X^{*}$; then $X^{*}$ is stable. Furthermore, if $L$ also satisfies

3. $\dot{L}<0$ in $\Omega-X^{*}$, then $X^{*}$ is asymptotically stable,

It then requires we show that $Z^{*}$ is an asymptotically stable point for $\dot{Z}=\varphi(Z)$ if $\varphi(Z)$ is continuously differentiable and the original of the linearized system

$$
\dot{y}=\varphi_{Z}\left(Z^{*}\right) y,
$$

where $y:=Z-Z^{*}$, is exponentially stable (i.e. all eigenvalues of $\varphi_{Z}\left(Z^{*}\right)$ are strictly negative). It was however noted that at $Z^{*},\left(Z^{*}, \lambda^{0}\left(Z^{*}\right)\right)$ satisfies eqns. (18) and (19) because the right hand side of eqn. (24) is identically zero; implying that $\lambda^{*}=$ $\lambda^{0}\left(Z^{*}\right)$.

Applying Taylor's expansion to the RHS of eqn. (36), about the point $Z^{*}$, gives the following linearized equation

$$
\frac{d Z}{d t}=\varphi(Z)+\varphi_{Z}(Z) y .
$$

Comparing eqn. (38) with the RHS of eqn. (23) yields

$$
\frac{d Z}{d t}=\varphi\left(Z^{*}\right)+\varphi_{Z}\left(Z^{*}\right)\left(Z-Z^{*}\right) .
$$

Recall that

$$
\begin{aligned}
L(Z, \lambda) & =J(Z)+\lambda^{T} g(Z), \\
L_{Z}(Z, \lambda) & =J_{Z}(Z)+\lambda^{T} g_{Z}(Z), \\
L_{Z Z}(Z, \lambda) & =J_{Z Z}(Z)+g_{Z Z}^{T}(Z) \lambda,
\end{aligned}
$$

then from eqn. (38),

$$
\frac{d Z}{d t}=\varphi\left(Z^{*}\right)+\varphi_{Z}\left(Z^{*}\right) y, \quad\left[\text { where } y=Z-Z^{*}\right] .
$$

Suppose $\dot{\varphi}\left(Z^{*}\right)=L_{Z}(Z, \lambda)$ which is equal to eqn. (41); then differentiating eqn. (41) arrives at

$$
\begin{aligned}
\varphi_{Z}\left(Z^{*}\right) & =\left[J_{Z Z}\left(Z^{*}\right)+\lambda^{T} g_{Z Z}\left(Z^{*}\right)+g_{Z}\left(Z^{*}\right) \lambda_{Z}\right] y, \\
& \left.=\left[L_{Z Z}\left(Z^{*}, \lambda^{*}\right)\right)+g_{Z}\left(Z^{*}\right) \lambda_{Z}\right] y .
\end{aligned}
$$

Therefore, eqn. (39) becomes

$$
\begin{aligned}
\frac{d Z}{d t} & =-\left[L_{Z}\left(Z^{*}, \lambda^{*}\right)+\left[L_{Z Z}\left(Z^{*}, \lambda^{*}\right)\right.\right. \\
& \left.\left.+g_{Z}^{T}\left(Z^{*}\right) \lambda_{Z}^{0}\left(Z^{*}\right)\right]\left(Z-Z^{*}\right)\right],
\end{aligned}
$$

where $\varphi\left(Z^{*}\right)=L_{Z}\left(Z^{*}, \lambda^{*}\right)$ and $\varphi_{Z}\left(Z^{*}\right)=$ $L_{Z Z}\left(Z^{*}, \lambda^{*}\right)+g_{Z}^{T}\left(Z^{*}\right) \lambda_{Z}^{0}\left(Z^{*}\right)$.

Considering the unknown coefficient $\lambda_{Z}^{0}\left(Z^{*}\right)$ and setting $L_{Z}\left(Z^{*}, \lambda^{*}\right)=$ 0 yields,

$$
\left.\frac{d Z}{d t}=-\left[L_{Z Z}\left(Z^{*}, \lambda^{*}\right)+g_{Z}^{T}\left(Z^{*}\right) \lambda_{Z}^{0}\left(Z^{*}\right)\right]\left(Z-Z^{*}\right)\right] .
$$

We seek to differentiate both sides of eqn. (32) with respect to $Z$ and evaluating at $Z^{*}$. Firstly, we differentiate the LHS of eqn. (32) as follows:

$$
\begin{aligned}
& g_{Z}\left(Z^{*}\right) \frac{d}{d Z}\left[J_{Z}(Z)+\lambda^{0}(Z) g_{Z}^{T}(Z)\right] \\
& +\left[J_{Z}(Z)+\lambda^{0}(Z) g_{Z}^{T}(Z)\right] \frac{d}{d Z} g_{Z}(Z) \\
& =g_{Z}\left(Z^{*}\right)\left[J_{Z Z}\left(Z^{*}\right)+\lambda_{0}\left(Z^{*}\right) \frac{d}{d Z} g_{Z}^{T}\left(Z^{*}\right)\right. \\
& \left.+g_{Z}^{T}(Z) \frac{d}{d Z} \lambda^{0}(Z)\right] \\
& +\left[J_{Z}\left(Z^{*}\right) g_{Z Z}\left(Z^{*}\right) \lambda^{0}(Z) g_{Z}^{T}\left(Z^{*}\right) g_{Z Z}\left(Z^{*}\right)\right.
\end{aligned}
$$

and arrives at

$$
\begin{aligned}
& {\left[J_{Z Z}\left(Z^{*}\right) g_{Z}\left(Z^{*}\right)+\lambda^{0} g_{Z Z}^{T}\left(Z^{*}\right)\right] g_{Z}\left(Z^{*}\right) } \\
& +\left[J_{Z}\left(Z^{*}\right)\right. \\
+ & \left.\lambda_{Z}^{0}\left(Z^{*}\right) g_{Z}^{T}\left(Z^{*}\right)\right] g_{Z Z}\left(Z^{*}\right) \\
+ & \lambda_{Z}^{0}\left(Z^{*}\right) g_{Z}^{T}\left(Z^{*}\right) g_{Z}\left(Z^{*}\right) .
\end{aligned}
$$

Secondly, we differentiate the RHS of eqn. (32) as follows;

$$
\begin{array}{cc} 
& \tau \frac{d}{d Z}\left[g_{Z}^{T}(Z) g_{Z}(Z) g(Z)\right] \\
= & \tau\left[g_{Z}^{T}(Z) \frac{d}{d Z} g_{Z}(Z) g(Z)\right. \\
& \left.+g_{Z}(Z) g(Z) \frac{d}{d Z} g_{Z}^{T}(Z)\right], \\
= & \tau\left[g_{Z}^{T}\left(Z^{*}\right) g_{Z}\left(Z^{*}\right)\right] g_{Z}\left(Z^{*}\right) \\
& +\tau\left[g_{Z}^{T}\left(Z^{*}\right) g_{Z Z}\left(Z^{*}\right)\right] g\left(Z^{*}\right) \\
+ & \tau\left[g_{Z Z}^{T}\left(Z^{*}\right)\right] g_{Z}\left(Z^{*}\right) g\left(Z^{*}\right) .
\end{array}
$$

Equating eqns. (48) and (49), we have

$$
\begin{aligned}
& {\left[J_{Z Z}\left(Z^{*}\right) g_{Z}\left(Z^{*}\right)+\lambda^{0} g_{Z Z}^{T}\left(Z^{*}\right)\right] g_{Z}\left(Z^{*}\right)+\left[J_{Z}\left(Z^{*}\right)\right.} \\
& \left.+\lambda_{Z}^{0}\left(Z^{*}\right) g_{Z}^{T}\left(Z^{*}\right)\right] g_{Z Z}\left(Z^{*}\right)+\lambda_{Z}^{0}\left(Z^{*}\right) g_{Z}^{T}\left(Z^{*}\right) g_{Z}\left(Z^{*}\right) \\
& =\tau\left[g_{Z}^{T}\left(Z^{*}\right) g_{Z}\left(Z^{*}\right)\right] g_{Z}\left(Z^{*}\right) \\
& +\tau\left[g_{Z}^{T}\left(Z^{*}\right) g_{Z Z}\left(Z^{*}\right)\right] g\left(Z^{*}\right) \\
& +\tau\left[g_{Z Z}^{T}\left(Z^{*}\right)\right] g_{Z}\left(Z^{*}\right) g\left(Z^{*}\right) .
\end{aligned}
$$


Applying eqns. (18) and (19) on eqn. (51), we have

$$
\begin{aligned}
& g_{Z}\left(Z^{*}\right)\left[L_{Z Z}\left(Z^{*}, \lambda^{*}\right)+g_{Z}^{T}\left(Z^{*}\right) \lambda_{Z}^{0}\left(Z^{*}\right)\right] \\
& =\tau g_{Z}\left(Z^{*}\right) g_{Z}^{T}\left(Z^{*}\right) g_{Z}\left(Z^{*}\right)
\end{aligned}
$$

where $L_{Z Z}\left(Z^{*}, \lambda^{*}\right)=J_{Z Z}\left(Z^{*}\right) g_{Z}\left(Z^{*}\right)+\lambda^{0} g_{Z Z}^{T}\left(Z^{*}\right)$ and $L_{Z}\left(Z^{*}, \lambda\right)=$ $\left.J_{Z}\left(Z^{*}\right)+\lambda_{Z}^{0}\left(Z^{*}\right) g_{Z}^{T}\left(Z^{*}\right)\right)$.

Eqn. (52) becomes

$$
\begin{aligned}
& g_{Z}\left(Z^{*}\right) L_{Z Z}\left(Z^{*}, \lambda^{*}\right)+G\left(Z^{*}\right) \lambda_{Z}^{0}\left(Z^{*}\right) \\
& =\tau G\left(Z^{*}\right) g_{Z}\left(Z^{*}\right),
\end{aligned}
$$

where $G\left(Z^{*}\right)=g_{Z}^{T}\left(Z^{*}\right) g_{Z}\left(Z^{*}\right)$.

Making $\lambda_{Z}^{0}\left(Z^{*}\right)$ the subject of eqn. (53) yields

$$
\lambda_{Z}^{0}\left(Z^{*}\right)=-G^{-1}\left(Z^{*}\right) g_{Z}\left(Z^{*}\right) L_{Z Z}\left(Z^{*}, \lambda^{*}\right)+\tau g_{Z}\left(Z^{*}\right) .
$$

Recall that

$$
\begin{aligned}
y & =Z-Z^{*}, \\
\Rightarrow \quad \frac{d y}{d t} & =\frac{d Z}{d t}-\frac{d Z^{*}}{d t}, \\
\text { and } \quad \frac{d y}{d t} & =\frac{d Z}{d t} .
\end{aligned}
$$

Substituting eqn. (54) into eqn. (46) and considering eqn. (57) yields

$$
\begin{aligned}
\frac{d y}{d t}= & -\left[L_{Z Z}\left(Z^{*}, \lambda^{*}\right)+\right. \\
& g_{Z}^{T}\left(Z^{*}\right)\left(-G^{-1}\left(Z^{*}\right) g_{Z}\left(Z^{*}\right) L_{Z Z}\left(Z^{*}, \lambda^{*}\right)\right. \\
& \left.\left.+\tau g_{Z}\left(Z^{*}\right)\right)\right] y, \\
\frac{d y}{d t}=\quad & -\left[L_{Z Z}\left(Z^{*}, \lambda^{*}\right)+\right. \\
& +g_{Z}^{T}\left(Z^{*}\right) g_{z}\left(Z^{*}\right) L_{Z Z}\left(Z^{*}, \lambda^{*}\right) G^{-1}\left(Z^{*}\right) \\
& \left.-\tau g_{Z}\left(Z^{*}\right) g_{Z}^{T}\left(Z^{*}\right)\right] y, \\
\frac{d y}{d t=} & {\left[-L_{Z Z}\left(Z^{*}, \lambda^{*}\right)\left[I-g_{Z}^{T}(Z) G^{-1}(Z) g_{Z}(Z)\right]\right.} \\
& \left.-\tau g_{Z}^{T}(Z) g_{Z}(Z)\right] y .
\end{aligned}
$$

Since $Z^{*}$ is zero at equilibrium point, then

$$
\begin{aligned}
\frac{d y}{d t} & =-\left[\left(I-g_{Z}^{T}(Z) G^{-1}(Z) g_{Z}(Z)\right) L_{Z Z}\left(Z^{*}, \lambda^{*}\right)\right. \\
& \left.+\tau g_{Z}^{T}(Z) g_{Z}(Z)\right] y .
\end{aligned}
$$

Let

$$
\begin{aligned}
S(Z) & =I-g_{Z}^{T}(Z) G^{-1}(Z) g_{Z}(Z), \\
V(Z) & =g_{Z}(Z) g_{Z}^{T}(Z),
\end{aligned}
$$

where $I$ is the $N \times N$ identity matrix. Therefore, eqn. (61) becomes

$$
\frac{d y}{d t}=-\left[S\left(Z^{*}\right) L_{Z Z}\left(Z^{*}, \lambda^{*}\right)+\tau V\left(Z^{*}\right)\right] y .
$$

From above, eqns. (62) and (63) are orthogonal where eqn. (62) is an operator which projects a vector to the null space of $g_{Z}(Z)$, because $g_{Z}(Z) S(Z)=0$.

Multiplying eqn. (64) from the left by $g_{Z}\left(Z^{*}\right)$ yields

$$
g_{Z}\left(Z^{*}\right) \frac{d y}{d t}=-\left[\tau g_{Z}\left(Z^{*}\right) V\left(Z^{*}\right)\right] y .
$$

Eqn. (65) is equivalent to

$$
\frac{d y}{d t}=-\left[\tau V\left(Z^{*}\right)\right] y \text {. }
$$

Hence, since $\tau>0$, it remains to show that $V\left(Z^{*}\right)$ is positive definite. Recall that $V\left(Z^{*}\right)=g_{Z}^{T}\left(Z^{*}\right) g_{Z}\left(Z^{*}\right)$ and $g_{Z}\left(Z^{*}\right)=G$; where $G$ whose dimension is $N \times(2 N+1)$ is given by eqn. (3.3). Therefore, $Q=G G^{T}$ with dimension $N \times N$ is given by

$$
Q=\left[\begin{array}{ccccc}
1+d^{2} & -c & 0 & \cdots & 0 \\
-c & \alpha & -c & 0 & \vdots \\
0 & \ddots & \ddots & \ddots & 0 \\
\vdots & \ddots & \ddots & \ddots & -c \\
0 & \cdots & 0 & -c & \alpha
\end{array}\right],
$$

where $\alpha=1+c^{2}+d^{2}$.

Theorem 3.4 (Sylvester's Criterion). A real, symmetric matrix is positive definite if and only if all the principal minors are positive definite. Gilbert [7].

Considering the implementation of Theorem 3.4 on the matrix $Q$, we seek to establish that the matrix is positive definite. Firstly, $Q$ is real since for every $q_{i, j} \in Q, q_{i, j} \in R$ (i.e. all the diagonal entries in eqn. (67) are real).

Secondly, $Q=g_{Z}^{T}\left(Z^{*}\right) g_{Z}\left(Z^{*}\right)$ is symmetric since $(Q)^{T}=\left[g_{Z}^{T}\left(Z^{*}\right) g_{Z}\left(Z^{*}\right)\right]^{T}$ $g_{Z}^{T}\left(Z^{*}\right) g_{Z}\left(Z^{*}\right)=Q$.

However, we also seek to establish that the principal minors $Q_{i}$, $\forall i=1,2,3, \ldots N$, of $Q$ are all positive definite. When $i=1,2,3$, and $4, Q_{1}, Q_{2}, Q_{3}$ and $Q_{4}$ are $1,1+c^{2}, 1+c^{2}+c^{4}$ and 1 respectively; and are all positive $\forall c, d>0$. We then assumed, by mathematical induction, that it is true for $Q_{k}$ when $i=k$ and $Q_{k+1}$ when $i=k+1$. And by Cholesky, it was shown that $Q_{k+1}$ is positive definite and that all the principal minors of $Q$ are positive definite [See Appendix]. We, therefore, concluded that the matrix $Q=G G^{T}$ is positive definite.

\subsection{Construction of the Modified Gradient Flow Method (MGFM)}

We now considered the discretization of the formulation of eqns. (23) and (24) using three level implicit time discretization scheme with splitting parameters $\theta_{1}$ and $\theta_{2}$ where $\theta_{3}=\left(1-\theta_{1}-\right.$ $\theta_{2}$ ).

Let $0=t_{0}<t_{1}<t_{2}<t_{3}<\ldots<t_{k}=t$ be a sequence time points for the time $t \geq t_{0}, h_{k}=t_{k+1}-t_{k}$ and $\delta_{Z_{k+1}}=Z_{k+2}-Z_{k}$ being the sequence of solution distance between two successive 
equilibrium points. We discretize eqn. (23) by the following implicit time stepping algorithm;

$$
\begin{aligned}
& \frac{Z_{k+2}-Z_{k+1}}{h_{k+1}}=-\left[\theta_{3} L_{Z}\left(Z_{k+2}, \lambda_{k+2}^{0}\right)+\right. \\
& \left.\theta_{2} L_{Z}\left(Z_{k+1}, \lambda_{k+1}^{0}\right)+\theta_{1} L_{Z}\left(Z_{k}, \lambda_{k}^{0}\right)\right] .
\end{aligned}
$$

Simplified to

$$
\begin{aligned}
& Z_{k+2}-Z_{k+1}=-h_{k+1}\left[\theta_{3} L_{Z}\left(Z_{k+2}, \lambda_{k+2}^{0}\right)+\right. \\
& \left.\theta_{2} L_{Z}\left(Z_{k+1}, \lambda_{k+1}^{0}\right)+\theta_{1} L_{Z}\left(Z_{k}, \lambda_{k}^{0}\right)\right] .
\end{aligned}
$$

Employing Taylor's expansion about $\left(Z_{k+2}, \lambda_{k+2}^{0}\right)$ arrives at

$$
\begin{aligned}
Z_{k+1}= & Z_{k}+h_{k} Z_{k}+O\left\|h_{k}\right\|^{2}, \\
L_{Z}\left(Z_{k+2}, \lambda_{k+2}^{0}\right)= & L_{Z}\left(Z_{k}, \lambda_{k}^{0}\right)+H\left(Z_{k}, \lambda_{k}^{0}\right) \delta_{Z_{k+1}} \\
& +O\left\|\delta_{Z_{k+1}}\right\|^{3}, \\
L_{Z}\left(Z_{k+1}, \lambda_{k+1}^{0}\right)= & L_{Z}\left(Z_{k}, \lambda_{k}^{0}\right)+H\left(Z_{k}, \lambda_{k}^{0}\right) \delta_{Z_{k}} \\
& +O\left\|\delta_{Z_{k}}\right\|^{3},
\end{aligned}
$$

where, $\delta_{Z_{k}}=Z_{k+1}-Z_{k}$ and

$H\left(Z_{k}, \lambda_{k}\right)=L_{Z Z}\left(Z_{k}, \lambda_{k}^{0}\right)+L_{Z \lambda_{k}^{0}}\left(\left(Z_{k}, \lambda_{k}\right)\right) \lambda_{Z}\left(Z_{k}\right)$

is the Hessian of $L(Z, \lambda)$. Substituting eqns. (70), (71) and (72) into eqn. (69) yields

$$
\begin{aligned}
Z_{k+2}= & Z_{k}+h_{k} Z_{k}^{\prime}+O\left\|h_{k}\right\|^{2} \\
& -h_{k+1}\left[\theta _ { 3 } \left(L_{Z}\left(Z_{k}, \lambda_{k}^{0}\right)\right.\right. \\
& \left.+H\left(Z_{k}, \lambda_{k}^{0}\right) \delta_{Z_{k+1}}+O\left\|\delta_{Z_{k+1}}\right\|^{3}\right) \\
& +\theta_{2}\left(L_{Z}\left(Z_{k}, \lambda_{k}^{0}\right)+H\left(Z_{k}, \lambda_{k}^{0}\right) \delta_{Z_{k}}\right. \\
& \left.\left.+O\left\|\delta_{Z_{k}}\right\|^{2}\right)+\theta_{1} L_{Z}\left(Z_{k}, \lambda_{k}^{0}\right)\right] .
\end{aligned}
$$

Neglecting the higher order terms in eqn. (73) and solving the resultant equation for $Z_{k+2}$ yields

$$
\begin{aligned}
Z_{k+2}= & Z_{k}+h_{k} Z_{k}-h_{k+1} \theta_{3} L_{Z k} \\
& -h_{k+1} \theta_{3} H_{Z k} Z_{k+2}+h_{k+1} \theta_{3} H_{Z k} Z_{k} \\
& -h_{k+1} \theta_{2} L_{Z k}++h_{k+1} h_{k} \theta_{2} H_{Z k} L_{Z_{k}} \\
& -h_{k+1} \theta_{1} L_{Z_{k}} .
\end{aligned}
$$

For equal step size, $h_{k+1}=h_{k}$ with $\theta_{1}=0$ we obtain $Z_{k+2}$ expressed below

$$
\begin{aligned}
Z_{k+2}= & Z_{k}+h_{k} Z_{k}-h_{k} \theta_{3} L_{Z k}-h_{k} \theta_{3} H_{Z k} Z_{k+2} \\
& +h_{k} \theta_{3} H_{Z k} Z_{k}-h_{k} \theta_{2} L_{Z k} \\
& +h_{k}^{2} \theta_{2} H_{Z k} L_{Z_{k}} .
\end{aligned}
$$

In collecting like terms and factorizing eqn. (75), it becomes

$$
\begin{aligned}
(I+ & \left.h_{k} \theta_{3} H_{Z k}\right) Z_{k+2} \\
& =\left(I+h_{k} \theta_{3} H_{Z_{k}}\right) Z_{k}-\left(\theta_{2}+\theta_{3}\right) h_{k} L_{Z k} \\
& +h_{k}^{2} \theta_{2} H_{Z k} L_{Z k}, \\
& =\left(I+h_{k} \theta_{3} H_{Z_{k}}\right) Z_{k}+\left(-h_{k} I\right. \\
& \left.+h_{k}^{2} \theta_{2} H_{Z k}\right) L_{Z k} .
\end{aligned}
$$

Making $Z_{k+2}$ the subject of formula in eqn. (76) yields

$$
\begin{aligned}
Z_{k+2}= & \left(I+h_{k} \theta_{3} H_{Z k}\right)^{-1}\left[\left(I+h_{k} \theta_{3} H_{Z k}\right) Z_{k}\right. \\
& \left.+\left(-h_{k} I+h_{k}^{2} \theta_{2} H_{Z k}\right) L_{Z k}\right], \\
= & Z_{k}-h_{k}\left(I+h_{k} \theta_{3} H_{Z k}\right)^{-1} \\
& \times\left[I-h_{k} \theta_{2} H_{Z k}\right] L_{Z_{k}},
\end{aligned}
$$

for any $\theta_{2}, \theta_{3} \in[0,1]$ and $\theta_{2}+\theta_{3}=1$ by the time-stepping algorithm for convex functions.

Also, from eqn. (76), we obtained

$$
\begin{aligned}
& \left(I+h_{k} \theta_{3} H_{Z_{k}}\right)\left(Z_{k+2}-Z_{k}\right) \\
& =-h_{k}\left(I+h_{k} \theta_{2} H_{Z k}\right) L_{Z k} .
\end{aligned}
$$

Given that $\delta_{k+1}=Z_{k+2}-Z_{k}$ and $\theta_{2}=0$, eqn. (77) becomes

$$
\left(I+h_{k} \theta_{3} H_{Z_{k}}\right) \delta_{Z_{k+1}}=-h_{k} L_{Z k} .
$$

Thus, for any initial guess $Z_{0}$, eqn. (77) defines a series converging to the solution of the optimal control problem stated in eqns. (1) to (3). Hence, the Modified Gradient Flow (MGF) Algorithm for the Discretized Optimal Control Problem (OCP) is stated as follows:

\begin{tabular}{l}
\hline MGF Algorithm for Discretized OCP \\
\hline \hline Step 1: Input the operator $G \in R^{N \times(2 N+1)}$, \\
$Z_{0} \in R^{2 N+1}$, parameters $\theta_{2}, \theta_{3} \in[0,1]$, \\
sequence of time step-size $\left\{h_{k}\right\}$ and tolerance \\
$(\sigma>0)$ sufficiently small. Set $k=0$. \\
Step 2: Compute $\lambda_{Z_{k}}^{0}$ using eqn. (54). \\
Step 3: Solve for $\delta_{Z_{k+1}}$ in the system using \\
the CGM $\left(I+h_{k} \theta_{3} H_{Z_{k}}\right) \delta_{Z_{k+1}}=-h_{k} L_{Z_{k}}$ \\
(where $\left.\delta_{Z_{k+1}}=Z_{k+2}-Z_{k}\right)$ \\
Step 4: Update: $Z_{k+2}=Z_{k}+\delta_{Z_{k+1}}$. \\
Step 5: Stop the process if $\left\|H_{Z_{k}}\right\| \leq \sigma$, \\
otherwise, set $k=k+1$ and repeat step 3.
\end{tabular}

\subsection{Error and Convergence Analysis}

The convergence analyses of the MGFM requires us to shown the rate and type of convergence of the algorithm for varying values of parameters $\theta 1, \theta 2$ and $\theta 3$. We shall show that the algorithm is super-linearly convergent for some $\theta_{2}$ and $\theta_{3}$ and quadratically convergent if $\theta_{1}=\theta_{2}=0$ and $\theta_{3}=1$.

Theorem 3.5. Let $\left\{Z_{k}\right\}$ be the sequence defined in eqn. (77) and $\left(Z^{\mathfrak{a}}, \lambda^{a}\right)$ be the solution of eqns. (1) and (2). If the initial guess $Z_{0}$ is sufficiently close to $Z$, then we have

1. $Z_{k}$ converges linearly to $Z^{\mathfrak{a}}$ if $\theta_{1}, \theta_{2}, \theta_{3} \in[0,1]$ and $h_{k}>0$ is sufficiently small;

2. $Z_{k}$ converges to $Z^{a}$ quadratically if $\theta_{2}=0, \theta_{3}=1$ and $h_{k} \rightarrow \infty$.

We shall prove the two cases separately. 
- Case 1. $\theta_{1}, \theta_{2}, \theta_{3} \in[0,1]$.

From eqn. (77) we have: $\left(I+h_{k} \theta_{3} H_{Z_{k}}\right)\left(Z_{k+2}-Z_{k}\right)=$ $-h_{k}\left(I-h_{k} \theta_{2} H_{Z_{k}}\right) L_{Z_{k}}$.

Hence,

$$
\begin{aligned}
Z_{k+2}= & Z_{k}-h_{k}\left[\left(I-h_{k} \theta_{2} H_{Z_{k}}\right) L_{Z_{k}}+\right. \\
& \left.\theta_{3} H_{Z_{k}}\left(Z_{k+2}-Z_{k}\right)\right] .
\end{aligned}
$$

Let $\left(Z^{*}, \lambda^{*}\right)$ be the minimum points of eqns. (16) and (17) and $e_{k+1}=Z_{k+1}-Z^{*}$.

Subtracting $Z^{*}$ from both sides of the above equation and noting that we have

$$
\begin{aligned}
e_{k+1}= & e_{k}-h_{k}\left[\left(I-h_{k} \theta_{2} H_{Z_{k}}\right)\left(L_{Z_{k}}-L_{Z_{k}^{*}}\right)\right. \\
& \left.+\theta_{3} H_{Z_{k}}\left(e_{k+1}-e_{k}\right)\right] .
\end{aligned}
$$

Thus, by the Mean Value Theorem,

$$
\begin{aligned}
e_{k+1}= & e_{k}-h_{k}\left[\left(I-h_{k} \theta_{2} H_{Z_{k}}\right) H_{Z_{k}}^{\zeta k} e_{k}\right. \\
& \left.+\theta_{3} H_{Z_{k}}\left(e_{k+1}-e_{k}\right)\right],
\end{aligned}
$$

where $\zeta_{k}$ denotes a point in the line segment connecting $Z_{k}$ and $Z^{*}$. Taking the norm of both sides, we have

$$
\left\|e_{k+1}\right\| \leq \gamma\left(Z_{k}, \lambda_{k}^{0}, \zeta_{k}, \theta_{1}, \theta_{2}, \theta_{3}, h_{k}\right)\left\|e_{k}\right\|,
$$

where

$$
\begin{aligned}
& \gamma\left(Z_{k}, \lambda_{k}^{0}, \zeta_{k}, \theta_{1}, \theta_{2}, \theta_{3}, h_{k}\right)= \\
& \left\|\left\{I-h_{k}\left(I+\theta_{3} H_{Z_{k}}\right)^{-1}\left(I-\theta_{2} H_{Z_{k}}\right) H_{Z_{k}}^{\zeta k}\right\}\right\| .
\end{aligned}
$$

From eqn. (81), we see that if

$\gamma\left(Z_{k}, \lambda_{k}^{0}, \zeta_{k}, \theta_{1}, \theta_{2}, \theta_{3}, h_{k}\right)<1$, then $e_{k}$ converges to zero linearly. To show this, we need to prove that $H_{Z_{k}}$ is positive definite. But by theorems and, $H_{Z_{k}}$ is positive definite. With these, when $Z_{k}$ is sufficiently close to $Z^{*}$, from eqn. (82), it is seen that if $\theta_{1}, \theta_{2}, \theta_{3} \in[0,1]$ and $h_{k}$ sufficiently small, we have

$$
\begin{aligned}
& \gamma\left(Z_{k}, \lambda_{k}^{0}, \zeta_{k}, \theta_{1}, \theta_{2}, \theta_{3}, h_{k}\right) \\
& \leq\left(1-\frac{h_{k}\left(1-h_{k} \theta_{2} \lambda_{\text {min }}^{k}\right) \lambda_{\text {min }}^{k}}{1+h_{k} \theta_{3} \lambda_{\text {max }}^{k}}\right)<1,
\end{aligned}
$$

where $\lambda_{\min }^{k}$ and $\lambda_{\max }^{k}$ denote the minimum and maximum eigenvalues of $H_{Z_{k}}$, respectively. Therefore, eqn. (81) implies that $\lim _{k \rightarrow \infty} e_{k}=0$ is linear. This proves that $Z_{k}$ converges linearly to $Z^{a}$ if $\theta_{1}, \theta_{2}, \theta_{3} \in[0,1]$ and $h_{k}>0$ is sufficiently small.

- Case 2. $\theta_{3}=1$.

From eqn. (77), when $\theta_{3}=1$ and $h_{k}=h$, we have

$$
\frac{Z_{k+2}-Z_{k}}{h}=-\left(L_{Z_{k}}+H_{Z_{k}} \delta_{Z_{k+1}}\right) \text {. }
$$

When $h \rightarrow \infty$, the above equation becomes

$$
H_{Z_{k}} \delta_{Z_{k+1}}+L_{Z_{k}}=0 .
$$

Eqn. (84) coincides with the equation resulting from the application of the Newton's method to $L_{Z}=0$. Hence, $Z_{k}$ converges quadratically to $Z^{*}$ if $Z_{0}$ is sufficiently close to $Z^{*}$.

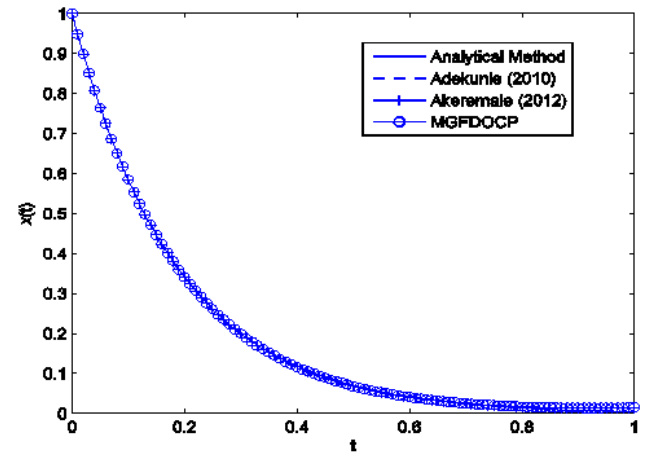

Figure 1. State Behaviors in various Algorithms

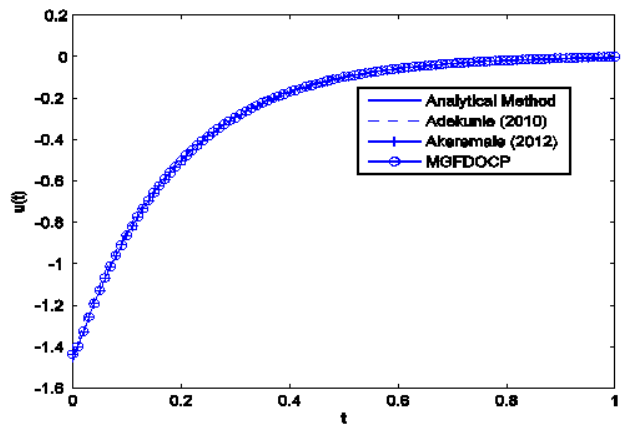

Figure 2. Control Behaviors in various Algorithms

\section{Examples and Numerical \\ Results}

The numerical examples and respective results are presented below based on the construction of modified gradient flow method (MGFM) for discretized optimal control problems. The results show the robustness and efficiency of the scheme (MGFM) as compared with existing schemes.

\section{Example 1}

Considering the optimal control problem presented by [1]

$$
\begin{array}{ll}
\text { Minimize } & I(x, u)=\int_{0}^{1}\left(x^{2}(t)+u^{2}(t)\right) d t \\
\text { subject to } & \dot{x}=2 x(t)+5 u(t), \quad x(0)=1 .
\end{array}
$$

The analytical solution is given as

$$
\left(\begin{array}{l}
x \\
\lambda
\end{array}\right)=\left(\begin{array}{c}
1.0000 \\
-0.5908
\end{array}\right) e^{-5.3852 t}
$$

and the control variable is given as $u(t)=$

$-1.4770 e^{-5.3852 t}$. The solution of eqns. (85) and (86) were obtained, using $h=0.1$ and $h_{k}=20$, by the modified gradient algorithm for discretized optimal control problems and the results were compared with the existing algorithms as shown in table 1 below. However, the step-size $h=0.1$ was chosen for the purpose of illustration. Meanwhile smaller choices of step-size could be used for better refinement. 


\begin{tabular}{cc}
\hline Algorithm & objective functional value \\
\hline \hline Analytical Method & 0.2953894 \\
\hline Adekunle [1] & 0.3033885 \\
\hline Akeremale [2] & 0.2955932 \\
\hline MGFM & 0.2955879 \\
\hline \hline
\end{tabular}

\begin{tabular}{cc}
\hline Algorithm & Objective Functional Value \\
\hline \hline Analytical Method & 0.5647 \\
\hline Adekunle [1] & 0.5746 \\
\hline Akeremale [2] & 0.5649 \\
\hline MGFM & 0.5648 \\
\hline \hline
\end{tabular}

Table 1: Comparison of Objective Functional Values of Existing Methods for Example 1

Results: From table 1 above, it was observed that the objective functional value (0.2955879) for the developed MGFM agreed favourably with the value $(0.2953894)$ obtained from the analytical method and better than the values obtained by Adekunle [1] and Akeremale [2] from Conjugate Gradient Method (CGM) and Extended Conjugate Gradient Method (ECGM) respectively.

Table 2a: Errors in the State variables for Example 1

\begin{tabular}{l|ccc}
\hline \hline \multirow{2}{*}{$\mathrm{t}$} & \multicolumn{3}{|c}{$|x(t)-\hat{x}(t)|\left(\times 10^{-3}\right)$} \\
\cline { 2 - 4 } & Adekunle [1] & Akeremale [2] & MGFM \\
\hline \hline 0.0 & 0 & 0 & 0 \\
0.1 & 3.2204 & 0.5528 & 0.5525 \\
0.2 & 3.8300 & 0.3680 & 0.3677 \\
0.3 & 3.4841 & 0.3414 & 0.3410 \\
0.4 & 2.9424 & 0.4447 & 0.4442 \\
0.5 & 2.5506 & 0.6968 & 0.6962 \\
0.6 & 2.4892 & 1.1667 & 1.1650 \\
0.7 & 2.9118 & 1.9867 & 1.9858 \\
0.8 & 4.0417 & 3.4014 & 3.4005 \\
0.9 & 6.2660 & 5.8292 & 5.8279 \\
1.0 & 10.266 & 9.9981 & 9.9789 \\
\hline \hline
\end{tabular}

\begin{tabular}{l|ccc}
\hline \hline \multirow{2}{*}{$\mathrm{t}$} & \multicolumn{3}{|c}{$|u(t)-\hat{u}(t)|\left(\times 10^{-4}\right)$} \\
\cline { 2 - 4 } & Adekunle [1] & Akeremale [2] & MGFM \\
\hline \hline 0.0 & 38.796 & 38.419 & 38.4202 \\
0.1 & 18.131 & 0.6668 & 0.6555 \\
0.2 & 7.9765 & 0.2654 & 0.2562 \\
0.3 & 3.1973 & 0.0149 & 0.0755 \\
0.4 & 1.1303 & 0.1891 & 0.1949 \\
0.5 & 4.3839 & 0.4248 & 0.4294 \\
0.6 & 4.8076 & 0.7726 & 0.7761 \\
0.7 & 1.0193 & 1.3417 & 1.3442 \\
0.8 & 2.0619 & 2.3045 & 2.3062 \\
0.9 & 3.8245 & 3.9497 & 3.9505 \\
1.0 & 6.7705 & 6.4026 & 6.4027 \\
\hline \hline
\end{tabular}

Table 2 above shows the errors in the state and control variables for example 1 while the errors generated with the MGFM were minimal compared with the errors generated from Adekunle [1] and Akeremale [2]. Figures 1 and $\mathbf{2}$ below clearly show the comparisons of the behaviors of the state and control trajectories to the various algorithms respectively. It was observed that the trajectories for both the state and control variables overlap due to closeness in results.

\section{Example 2}

Considering the optimal control problem presented by [1]

$$
\begin{gathered}
\operatorname{Minimize} J(x, u)=\int_{0}^{1}\left(x^{2}(t)+u^{2}(t)\right) d t, \\
\text { subject to } \quad \dot{x}=1.705 x(t)+3.021 u(t), \\
x(0)=1 .
\end{gathered}
$$

Table 3: Comparison of Objective Functional Values of Existing Methods for Example 2

Table 4a: Errors in the State variables for Example 1

\begin{tabular}{l|ccc}
\hline \hline \multirow{2}{*}{$\mathrm{t}$} & \multicolumn{3}{|c}{$|x(t)-\hat{x}(t)|\left(\times 10^{-4}\right)$} \\
\cline { 2 - 4 } & Adekunle [1] & Akeremale [2] & MGFM \\
\hline \hline 0.0 & 10.000 & 10.0000 & $1.0000 E$ \\
0.1 & 3.2204 & 5.528 & 5.5253 \\
0.2 & 2.0509 & 4.347 & 3.9394 \\
0.3 & 3.3242 & 2.604 & 2.3946 \\
0.4 & 3.2376 & 2.435 & 2.2688 \\
0.5 & 2.9852 & 2.646 & 2.4997 \\
0.6 & 2.6832 & 3.2433 & 3.0992 \\
0.7 & 2.3921 & 4.2860 & 4.1303 \\
0.8 & 2.1365 & 5.8933 & 5.7138 \\
0.9 & 1.9144 & 8.2567 & 8.0424 \\
1.0 & 1.7012 & 10.716 & 11.403 \\
\hline \hline
\end{tabular}

Table 4b: Errors in the Control variables for Example 1

Results: From table 3 above, it was observed that the objective functional value, 0.5648 , for MGFM agreed favourably with the value $(0.5647)$ obtained from the analytical method; and better than the values obtained by Adekunle [1] and Akeremale [2] from Conjugate Gradient Method (CGM) and Extended Conjugate Gradient Method (ECGM) respectively as expressed in table 3 above.

Results: Table 4 above shows the errors in the state and control variables for example 2 where the errors generated from the use of MGFM were minimal compared to the errors generated from same examples in Adekunle [1] and Akeremale [2]. Figures $\mathbf{3}$ and $\mathbf{4}$ below clearly show the comparisons of the behaviors of the state and control trajectories to the various algorithms 


\begin{tabular}{l|ccc}
\hline \hline \multirow{2}{*}{$\mathrm{t}$} & \multicolumn{3}{|c}{$|u(t)-\hat{u}(t)|\left(\times 10^{-4}\right)$} \\
\cline { 2 - 4 } & Adekunle [1] & Akeremale [2] & MGFM \\
\hline \hline 0.0 & 0.2928 & 0.3404 & 0.2893 \\
0.1 & 1.8337 & 0.5220 & 0.3179 \\
0.2 & 1.0622 & 1.8094 & 2.4019 \\
0.3 & 5.9872 & 1.1203 & 2.3334 \\
0.4 & 3.3442 & 0.4000 & 0.4941 \\
0.5 & 1.9971 & 0.7234 & 0.7965 \\
0.6 & 1.5137 & 1.1255 & 1.1823 \\
0.7 & 1.6411 & 1.6579 & 1.7019 \\
0.8 & 2.2516 & 2.3872 & 2.4210 \\
0.9 & 3.3101 & 3.4037 & 3.4311 \\
1.0 & 2.9285 & 3.4045 & 2.8932 \\
\hline \hline
\end{tabular}

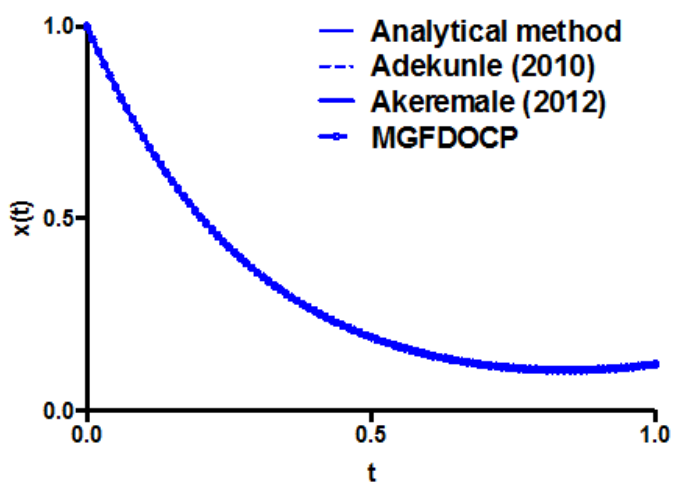

Figure 3. State Behaviors in various Algorithms

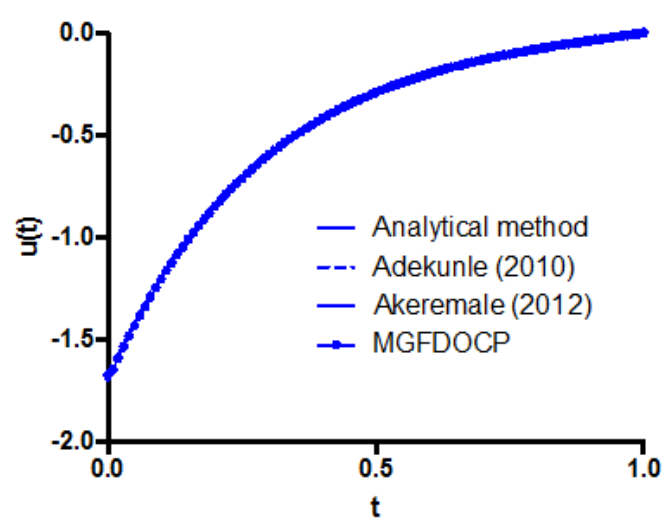

Figure 4. Control Behaviors in various Algorithms

respectively. It was observed that the trajectories for both the state and control variables overlap due to closeness in results.

\section{Conclusion}

This paper developed a new scheme for the solution of a quadratic optimal control problem with linear equality constraint. The method is a gradient flow formulation that requires dis- cretization techniques and a splitting parameter approach for the gradient of the equation whose solution converges to a local minimum of the original problem; either linearly or quadratically. Two linear equality constrained optimal control problems were considered and their results compared with the analytical and two known existing schemes. Thus, it was observed that the new scheme compared favorably with the analytic solutions and performed better than the Conjugate Gradient Method used in [2] in terms of convergence and accuracy. It is therefore obvious that this developed algorithm, due to its robustness and efficiency, would provide a new platform for solving optimal control problems with or without box constraints, equality or inequality constraints or with delay or non-delay dynamical systems.

\section{Acknowledgements}

The authors are grateful to the editors and the anonymous reviewers for their constructive comments and suggestions.

\section{References}

[1] A. I. Adekunle, "Algorithm for a Class of Discretized Optimal Control Problems", M.Tech. Thesis, Federal University of Technology, Akure, Nigeria (2011) (Unpublished).

[2] O. C. Akeremale, "Optimization of Quadratic Constrained Optimal Control Problems using Augumented Lagrangian Method", M.Tech. Thesis, Federal University of Technology, Akure, Nigeria (2012) (Unpublished)

[3] W. Behrman, "An Efficient Gradient Flow Method for Unconstrained Optimization", PhD Thesis, Stanford University (1998) (Unpublished).

[4] J. T. Betts, "Practical Methods for Optimal Control Problem Using Non linear programming", SIAM, Philadelphia, (2001).

[5] Y. Evtushenko, "Generalized Lagrange Multipliers Technique for Nonlinear Programming", JOTA, 21 (1977) 121.

[6] Y. G. Evtushenko \& V. G. Zhadan, "Stable Barrier Projection and Barrier Newton Methods", Nonlinear Programming Optimization Methods and Software, 3 (1994) 237.

[7] G. T. Gilbert, "Positive Definite Matrices and Sylvester's Criterion", The American Mathematical Monthly, Taylor \& Francis, 98 (1991) 44.

[8] W. M. Haddad, S. G. Nersesov \& V.S. Chellaboina, "Lyapunov Function Proof of Poincare's Theorem", International Journal of Systems Science, 35 (2004) 287.

[9] J. B. Layton, "Efficient direct computatioion of the Pseudo-inverse and its gradient", International Journal for Numerical Methods in Engineering, 40 (1997) 4211.

[10] W. H. Morris, S. Stephen \& L. D. Robert, "Differential Equations, Dynamical Systems, and an Introduction to Chaos", Elsevier Academic Press, USA (2004) 194.

[11] O. Olotu \& K. A. Dawodu, "Quasi-Newton Embedded Augmented Lagrangian Algorithm for Discretized Optimal Proportional Control Problems", Journal of Mathematical Theory and Modeling, 3 (2013a) 67.

[12] O. Olotu \& K. A. Dawodu, "On the Discretized Algorithm for Optimal Proportional Control Problems Constrained by Delay Differential Equations", Journal of Mathematical Theory and Modeling, 3 (2013b) 157.

[13] O. Olotu \& S. A. Olorunsola, "An Algorithm for a Discretized Constrained, Continuous Quadratic Control Problem", Journal of Applied Sciences, 8 (2006) 6249.

[14] L. S. Pontryagin, V. G. Boltyanskii, R. V. Gamkrelidze \& E. F. Mishchenko, "The Mathematical Theory of Optimal Processes", Interscience Publishers, London (1962).

[15] S. Wang, X. Q. Yang K. L. Teo "A Unified Gradient Flow Approach to Constrained Nonlinear Optimization Problems", Computational Optimization and Applications, 25 (2003) 251. 


\section{APPENDIX}

Let $Q_{i}$ represent the leading principal minor of $Q, \forall i=1,2,3, \ldots, N$ of the matrix $Q=G G^{T}$; we then considered the determinants of the principal minors $Q_{1}, Q_{2}, Q_{3}, \ldots$ as follows:

For $i=1, Q_{1}=1+d^{2}>0, \forall d$.

For $i=2$,

$$
Q_{2}=\left[\begin{array}{cc}
1+d^{2} & -c \\
-c & 1+c^{2}+d 2
\end{array}\right],
$$

such that $\operatorname{det}\left(Q_{2}\right)=1+2 d^{2}+c^{2} d^{2}+d^{4}>0$ for all $c, d$. For $i=3$,

$$
Q_{3}=\left[\begin{array}{ccc}
1+d^{2} & -c & 0 \\
-c & 1+c^{2}+d^{2} & -c \\
0 & -c & 1+c^{2}+d^{2}
\end{array}\right],
$$

such that $\operatorname{det}\left(Q_{3}\right)=1>0 \forall c$.

Therefore, $\left|Q_{3}\right|$ is positive.

For $i=4$,

$$
Q_{4}=\left[\begin{array}{cccc}
1+c^{2} & -c & 0 & -d \\
-c & 1+c^{2} & -c & 0 \\
0 & -c & 1 & 0 \\
-d & 0 & 0 & 2 d^{2}
\end{array}\right],
$$

such that det. $\left(Q_{4}\right)=d^{2}>0 . \forall c, d$.

Therefore, det $\left(Q_{4}\right)=d^{2}>0, \forall d$, i.e. $\left|Q_{4}\right|$ is positive. Hence, by mathematical induction, since it is true for values of $Q_{i}, i=$ $1,2,3,4$, then we assume that it is true for $i=k$, i.e. $Q_{k}$, while we shall prove that it is true for $i=k+1$, i.e. $Q_{k+1}$. Set $\sigma^{2}=$ $1+c^{2}>0, c^{2}>0,1>0,2 d^{2}>0$ and $d^{2}>0$ where $1+c^{2}, c^{2}$, $1,2 d^{2}$ and $d^{2}$ are principal diagonals of $Q$.

Thus,

$$
Q_{k+1}=\left[\begin{array}{cc}
Q_{k} & \beta_{k+1} \\
\beta_{k+1}^{T} & \sigma^{2}
\end{array}\right],
$$

where

$$
\beta_{k+1}=\left[\begin{array}{llll}
Q_{1, k+1} & Q_{2, k+1} & \ldots & Q_{k, k+1}
\end{array}\right]^{T} .
$$

By Cholesky, $Q_{k+1}$ is said to be positive definite if there exists a lower triangular matrix $L_{i, j}$ such that $Q_{k+1}=L L^{T}$. That is,

$$
\left[\begin{array}{cc}
Q_{k} & \beta_{k+1} \\
\beta_{k+1}^{T} & c^{2}
\end{array}\right]
$$

$$
=\left[\begin{array}{cc}
H_{k} & 0 \\
L_{k+1,1}^{T} & L_{k+1, k+1}
\end{array}\right]\left[\begin{array}{cc}
H_{k}^{T} & L_{k+1,1}^{T} \\
0 & L_{k+1, k+1}
\end{array}\right],
$$

where $H_{k}$ is a lower triangular matrix with positive diagonal entries. However, eqn. (5) becomes

$$
\left[\begin{array}{cc}
Q_{k} & \beta_{k+1} \\
\beta_{k+1}^{T} & c^{2}
\end{array}\right]=
$$

such that $\left[\begin{array}{cc}H_{k} H_{k}^{T} & H_{k} L_{k+1,1} \\ L_{k}^{T}={ }_{k}^{1,1} H_{k}^{T} & L_{k}^{T} H_{k}^{T+1} \beta_{k+1}^{T} \stackrel{L_{k+1,1}+L^{2}}{=} L_{k+1,1}^{T} H_{k}^{T}, \beta_{k+1}\end{array}\right]$, $\sigma^{2}=L_{k+1,1}^{T} L_{k+1,1}+L_{k+1}^{2} L_{k+1}$.

Thus, since the diagonal entries of $H_{k}$ are greater than zero, it is non-singular and therefore the linear system of the equation has a unique solution given by $L_{k+1,1}=H_{k}^{-1} \beta_{k+1}$ and a positive value for $L_{k+1, k+1}$ can be obtained if $\sigma^{2}-L_{k+1}^{T} L_{k+1}>0$. Hence,

$$
\begin{aligned}
0<\left|Q_{k}\right| & =\operatorname{det}\left[\begin{array}{cc}
Q_{k} & \beta_{k+1} \\
\beta_{k+1}^{T} & \sigma^{2}
\end{array}\right] \\
& =Q_{k}\left[\sigma^{2} I-\beta_{k+1}^{T}\left(Q_{k}\right)^{-1} \beta_{k+1}\right] .
\end{aligned}
$$

Substituting the values of $\beta_{k+1}$ and $Q_{k}$, we have

$$
\begin{aligned}
Q & =Q_{k}\left[\sigma^{2} I-\left(H_{k} L_{k+1,1}\right)^{T}\left(H_{k} H_{k}^{T}\right)^{-1} H_{k} L_{k+1,1}\right], \\
& =Q_{k} \sigma^{2}-L_{k+1,1}^{T} L_{k+1,1},
\end{aligned}
$$

where

$$
\begin{aligned}
\left(H_{k} H_{k}^{T}\right)^{-1} & =Q_{k}^{-1}, \\
Q_{k} & =\left[\sigma^{2}-L_{k+1,1}^{T} L_{k+1,1}\right] .
\end{aligned}
$$

Since $\operatorname{det} Q_{k}>0$, it follows that $\sigma^{2}-L_{k+1,1}^{T} L_{k+1,1}>0$. Hence,

$$
L_{k+1, k+1}=\sqrt{\sigma^{2}-L_{k+1,1}^{T} L_{k+1,1}}
$$

Thus $L_{i}>0$ arises from the fact that $G$ is positive definite (LICQ). Hence, the pair $\left(Z(t), \lambda^{0}(Z(t))\right)$ tends to $\left(Z^{*}, \lambda^{*}\right)$ as $t \rightarrow$ $\infty$ provided the starting point $Z_{0}$ is sufficiently close to point $Z^{*}$. 